\title{
Transcriptional upregulation of HER2 expression in the absence of $H E R 2$ gene amplification results in cetuximab resistance that is reversed by trastuzumab treatment
}

\author{
CRISTINA OLIVERAS-FERRAROS ${ }^{1,2}$, ANNA MASSAGUER VALL-LLOVERA ${ }^{3}$, \\ ALEJANDRO VAZQUEZ-MARTIN ${ }^{1,2}$, DOLORS CARRION SALIP ${ }^{3}$, BERNARDO QUERALT ${ }^{2,4}$, \\ SILVIA CUFI ${ }^{1,2}$, BEGOÑA MARTIN-CASTILLO ${ }^{2,5}$, JOAQUIM BOSCH-BARRERA ${ }^{2,4}$, \\ JOAN BRUNET $^{2,4}$, RAFAEL DE LLORENS ${ }^{3}$ and JAVIER A. MENENDEZ ${ }^{1,2}$ \\ ${ }^{1}$ Translational Research Laboratory, Catalan Institute of Oncology (ICO); ${ }^{2}$ Girona Biomedical Research Institute (IdIBGi);
${ }^{3}$ Biochemistry and Molecular Biology Unit, Department of Biology, University of Girona; ${ }^{4}$ Medical Oncology, Catalan
Institute of Oncology (ICO); ${ }^{5}$ Unit of Clinical Research, Catalan Institute of Oncology (ICO), Girona, Catalonia, Spain
}

Received January 24, 2012; Accepted February 28, 2012

DOI: $10.3892 /$ or.2012.1732

\begin{abstract}
The recent identification of HER2 gene amplification as a novel predictor of resistance to the EGFR (HER2)-targeted antibody cetuximab and of response to combination therapies against EGFR and HER2 in wild-type KRAS tumor settings may represent a further step toward personalized medicine for patients with colorectal cancer. Herein, we show that transcriptional upregulation of HER2 expression in the absence of HER2 gene amplification is a molecular phenomenon that takes place in EGFR-dependent, wild-type KRAS squamous cell carcinoma (SCC) cells that acquire resistance to cetuximab. Since cetuximab activity against cetuximab-refractory SCC cells can be fully restored in the presence of the antiHER2 monoclonal antibody trastuzumab, our findings suggest that, beyond HER 2 gene amplification, we might need to redefine the threshold values for HER2 positivity to improve the accuracy of the selection of cetuximab-refractory patients with wild-type KRAS that may benefit from receiving a cetuximab/ trastuzumab combination.
\end{abstract}

\section{Introduction}

Three recent studies aimed at delineating new mechanisms of therapeutic resistance to the anti-HER1 (EGFR) antibody cetuximab (Ctx; Erbitux $\left.{ }^{\circledR}\right)$ have concurrently identified aberrant HER2 (ERBB2) signaling as a novel mechanism for mediating Ctx

Correspondence to: Dr Javier A. Menendez, Catalan Institute of Oncology, Girona (ICO-Girona), Dr. Josep Trueta University Hospital, Avenida de França s/n, E-17007 Girona, Catalonia, Spain E-mail: jmenendez@iconcologia.net; jmenendez@idibgi.org

Key words: cetuximab, KRAS, EGFR, HER2, trastuzumab, lapatinib resistance in carcinomas with wild-type $K R A S$. Yonesaka et al (1) showed that activation of HER2 signaling in cultured cancer cells, through either HER2 gene amplification or upregulation of the HER2/HER3 ligand heregulin, is sufficient to cause Ctx resistance. Accordingly, colorectal cancer patients exhibiting either de novo or acquired resistance to $\mathrm{Ctx}$-based therapeutic regimens were found to have $H E R 2$ gene amplification or high levels of circulating heregulin. In their hands, HER2 inhibition or disruption of HER2/HER3 heterodimerization restored the antitumor activity of $\mathrm{Ctx}$ in vitro and in vivo. The suggestion of Yonesaka that a combination of HER2 inhibitor and Ctx treatment may represent a rational therapeutic strategy that should be assessed in colon cancer patients with Ctx-resistant carcinomas was also confirmed in a novel in vivo model of bladder carcinoma with acquired resistance to $\mathrm{Ctx}$.

Quesnelle and Grandis (2) showed that increased phosphorylation of a C-terminal fragment of HER2 mediates Ctx resistance both in vitro and in vivo because afatinib (BIBW-2992), an irreversible dual HER1/HER2 tyrosine kinase inhibitor (TKI), successfully inhibits the growth of Ctx-resistant carcinoma cells. Upon production of large xenograft cohorts from 85 patient-derived, genetically characterized metastatic colorectal cancer samples ('xenopatients'), Bertotti et al (3) generated genotype-Ctx response correlations indicating that $H E R 2$ gene amplification specifically occurs in a subset of Ctx-resistant, wild-type KRAS/NRAS/ $B R A F / P I K 3 C A$ cases. In agreement with Yonesaka et al (1), Bertotti et al (3) found a significant enrichment of HER2 gene amplification in clinically $\mathrm{Ctx}$-unresponsive wild-type $K R A S$ patients. Importantly, combined inhibition of EGFR and HER2 induced overt, long-lasting tumor regression in HER2 gene-amplified xenopatients. As discussed by Ciardiello and Normanno (4), the identification of HER2 as a predictor of resistance to the EGFR-targeted antibody Ctx and of response to combination therapies against EGFR and HER2 in wildtype KRAS tumor settings may represent a further step toward personalized medicine for patients with colorectal cancer. 
We now reveal that the acquisition of secondary resistance to $\mathrm{Ctx}$ in squamous cell carcinoma (SCC) cells is accompanied also by transcriptional upregulation of HER2 expression in absence of HER2 gene amplification, which is amenable to inhibition by HER2-targeted drugs. As the Ctx anti-tumoral activity was fully restored in the presence of the anti-HER2 monoclonal antibody trastuzumab, our current findings suggest that we need to redefine the threshold values for HER2 positivity beyond the sole presence of HER2 gene amplification to improving the accuracy of the selection of Ctx-refractory patients with wildtype $K R A S$ that might benefit from receiving a $\mathrm{Ctx} /$ trastuzumab combination.

\section{Materials and methods}

Drugs. Dual HER1/HER2-TKI1 lapatinib (GW572016; Tykerb $^{\circledR}$ ) was kindly provided by GlaxoSmithKline (GSK), Corporate Environment, Health \& Safety (Brentford, Middlesex, UK). Stock solution of lapatinib (10 mmol/l) were prepared in DMSO and stored in aliquots in the dark at $-20^{\circ} \mathrm{C}$ until utilization. Cetuximab (Erbitux) and trastuzumab $\left(\right.$ Herceptin $^{\circledR}$ ) were kindly provided by Hospital Universitari de Girona Dr Josep Trueta Pharmacy (Girona, Spain). Cetuximab was solubilized with $10 \mathrm{mmol} / \mathrm{l} \mathrm{NaCl}$ of potassium phosphate buffer (PBS) pH 7.2 in bacteriostatic water for injection purposes (stock solution at $2 \mathrm{mg} / \mathrm{ml}$ ), stored at $4^{\circ} \mathrm{C}$ and used within 1 month. Trastuzumab was solubilized in bacteriostatic water for injection containing $1.1 \%$ benzyl alcohol (stock solution at $21 \mathrm{mg} / \mathrm{ml}$ ), stored at $4^{\circ} \mathrm{C}$ and used within 1 month. For experimental use, experimental agents were prepared fresh from stock solutions and were diluted with cell growth media immediately before use.

Cell viability. Concentration-effect curves were generated as a plot of the fraction of surviving cells versus drug concentration by using a colorimetric 3-(4,5-dimethylthiazol-2-yl)-2,5-diphenyl tetrazolium bromide (MTT) reduction assay as previously described (11).

Real-time quantitative PCR (Taqman) analysis. RNA was isolated using the Qiagen RNeasy Mini kit and the expression of HER1 (EGFR), HER2 and HER3 genes was quantified by realtime quantitative PCR or Taqman using $100 \mathrm{ng}$ of total RNA per reaction, as described previously $(10,20,21)$. The sequences of the primer/probe sets used for this analysis are: EGFR (HER1) 5'-GCCTTGAGTCATCTATTCAAGCAC-3' (F), 5'-TGCT ACTGTCATTCGCACCTG-3' (R), and 5'-FAM-AGCTCTG GCCACAACAGGGCATTTT-TAMRA-p-3' (P); HER2 5'-TCTGGACGTGCCAGTGTGAA-3' (F), 5'-TGCTCCCT GAGGACACATCA-3' (R), and 5'-FAM-CAGAAGGCCAA GTCCGCAGAAGCC-TAMRA-p-3' (P); HER3 5'-TTCTCT ACTCTACCATTGCCCAAC-3' (F), where $\mathrm{F}$ and $\mathrm{R}$ are the forward and reverse primers, respectively, and $\mathrm{P}$ is the Taqman probe (FAM as reporter and TAMRA as quencher). Taqman reaction was done in a standard 96-well plate format with ABI 7500 real-time quantitative PCR system. For data analysis, raw $\Delta C_{t}$ was first normalized to a housekeeping gene (i.e., TBT: TATA-Box Binding Protein) for each sample to get $\Delta C_{t}$. The normalized $\Delta C_{t}$ was then calibrated to control (untreated) samples to get $\Delta \Delta C_{t}$. In the final step of data analysis, the $\Delta \Delta C_{t}$ were converted to fold-changes $\left(2^{-\Delta \Delta C_{t}}\right)$ relative to untreated controls.

HER2-specific enzyme-linked immunosorbent assay (ELISA). Determination of HER 2 protein content was performed with a commercially available quantitative ELISA (Oncogene Science, Bayer Diagnostics) according to the manufacturer's protocol. The concentrations of HER2 in test samples (in nanograms of HER 2 per milligram of total protein) were determined by interpolation of the sample absorbances from the standard curve. Each experiment was performed in duplicate wells.

Establishment of long-term accommodation to Ctx in EGFR gene-amplified, wild-type KRAS A431 SCC cells. Over a minimum period of 6 months, cultures of A431 vulvar squamous carcinoma cells (originally obtained from the American Type Culture Collection, Manassas, VA, USA) were continuously exposed to increasing concentrations of Ctx. A431 epidermoid carcinoma cells were chosen based on two primary criteria: (1) these EGFR-overexpressing cells are exquisitely sensitive to CTX; and (2) A431 cells have neither EGFR Tyrosine Kinase Domain or KRAS mutations. Commencing with the Inhibitory Concentration $50\left(\mathrm{IC}_{50}\right)$ of $\mathrm{Ctx}(\sim 25 \mu \mathrm{g} / \mathrm{ml})$, the exposure dose was progressively increased 2-3 weeks until four dose doubling had been successfully achieved. Controlled parental cells were cultured in parallel and exposed to the PBS vehicle. We established two Long Term (LT)-Ctx adapted A431 pooled populations that were then maintained in continuous culture with the maximal achieved dose of Ctx. When challenged to Ctx doses as high as $200 \mu \mathrm{g} / \mathrm{ml}$, they retained a $>90 \%$ active metabolic status (as assessed by MTT-based cell viability assays) compared to Ctx-naïve A431 parental cells (which decreased their ability to metabolize MTT by $\sim 70-80 \%$ following treatment with an equivalent dose of Ctx).

Parental A431 cells and LT-Ctx adapted pools were routinely grown in Dulbecco's modified Eagle's medium (DMEM, Gibco ${ }^{\circledR}$ Cell Culture Systems, Invitrogen S.A., Barcelona, Spain) containing 10\% heat-inactivated fetal bovine serum (FBS, Bio-Whittaker, Inc., Walkersville, MD, USA), $1 \%$ L-glutamine, $1 \%$ sodium pyruvate, $50 \mathrm{U} / \mathrm{ml}$ penicillin, and $50 \mu \mathrm{g} / \mathrm{ml}$ streptomycin (culture media for LT-Ctx adapted pools included $100 \mu \mathrm{g} / \mathrm{ml} \mathrm{Ctx}$, which was freshly added during culture splitting). Cells were maintained at $37^{\circ} \mathrm{C}$ in a humidified atmosphere of $95 \%$ air $/ 5 \% \mathrm{CO}_{2}$. Cells were screened periodically for Mycoplasma contamination.

Statistics. Two-group comparisons were performed by the Student $t$ test for paired and unpaired values. Comparisons of means of $\geq 3$ groups were performed by ANOVA, and the existence of individual differences, in case of significant $F$ values at ANOVA, tested by Scheffé's multiple comparisons. In all the cases statistical analyses were carried out with XLSTAT (Addinsoft $^{\mathrm{TM}}$ ) and $\mathrm{P}<0.01$ was considered to be significant.

\section{Results}

HER2 overexpression is sufficient to impede cetuximab functioning in EGFR-overexpressing cancer cells. To definitely test the hypothesis that HER2 upregulation is sufficient to cause Ctx resistance, we used MCF10A cells, a useful in vitro 

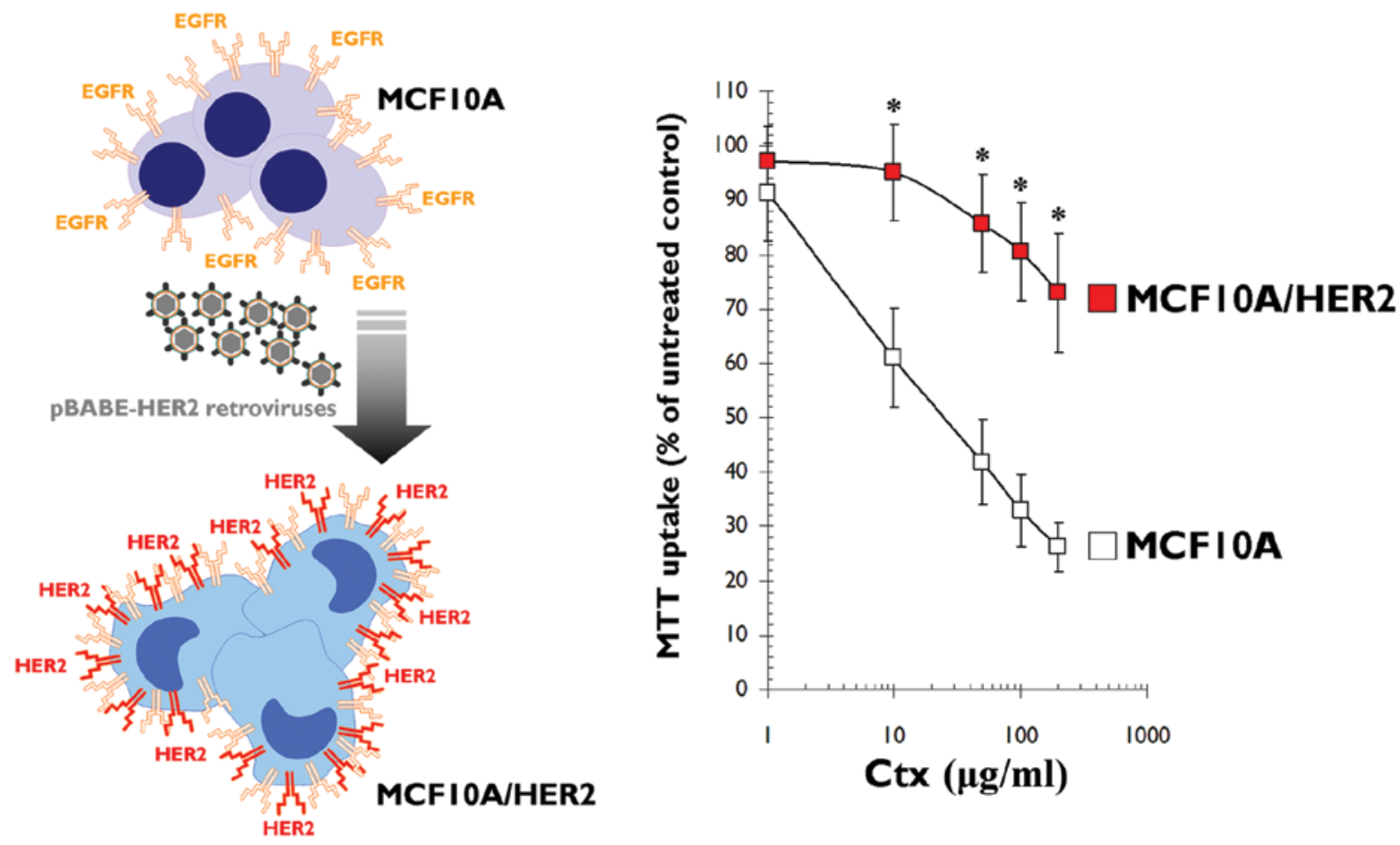

Figure 1. The sole overexpression of HER2 is sufficient to impede the Ctx function against EGFR-dependent cancer cells. MCF10A parental cells and their derivatives stably transduced with a pBABE-HER2 retroviral vector were cultured in the absence or presence of graded concentrations of Ctx for 5 days. Cell viability was measured with the MTT uptake assay and expressed as \% of untreated cell population $(=100 \%)$. Results are means and $95 \%$ confidence intervals (bars) of five independent experiments made in triplicate. Statistically significant differences (one-factor ANOVA analysis) between MTT uptakes in MCF10A and MCF10A/HER2 cells are shown $(" \mathrm{P}<0.01)$. Statistical tests were two-sided.

system for analyzing the effects of the expression of specific cancer- and chemoresistance-related genes (e.g., HER2) in a nontransformed, near diploid, spontaneously immortalized genetic background (5-7). In addition to naturally expressing very low levels of HER2, MCF10A cells constitutively express high levels of EGFR and are dependent on EGF for optimal growth. Hence, overexpression of HER2 in these cells recapitulates the common clinical situation of HER1 and HER2 co-overexpression, and with the addition of EGF, the paracrine/autocrine stimulation of HER receptors-driven oncogenic signaling is also recapitulated. MCF10A cells acquire both an in vitro transformed and an in vivo tumorigenic phenotype following transfection with appropriate vectors containing the HER2 gene (5-8).

We employed MCF10A cells that were engineered to overexpress the wild-type form of human HER 2 by retroviral infection, thus avoiding undesirable clone-related effects (Fig. 1, left). When HER2-specific ELISA assays were used to quantitatively measure the change in the concentration of the HER 2 protein following retroviral infection and stable selection, empty vector-transduced MCF10A/pBABE cells and MCF10A/HER2 cells were found to express $60 \mathrm{ng}$ of HER $2 \mathrm{mg}$ protein ${ }^{-1}$ and 2,200 $\mathrm{ng}$ of HER $2 \mathrm{mg}^{-1}$ protein, respectively (8). Of note, the retrovirally driven upregulation of HER2 in MCF10A/HER2 cells can be considered to constitute bona fide HER2 overexpression because the HER2 protein levels are comparable to or greater than those found in cancer cell lines that naturally exhibit HER2 gene amplification and HER2 protein overexpression [e.g., >900 HER2 $\mathrm{ng} / \mathrm{mg}$ protein in SKBR3 and BT-474 breast carcinoma cell lines (9)]. More importantly for this study, the half-maximal inhibitory concentration $\left(\mathrm{IC}_{50}\right)$ of $\mathrm{Ctx}$ was as low as $25 \mu \mathrm{g} /$ $\mathrm{ml}$ in the parental MCF10A cells (Fig. 1C, right). However, in equivalent dose-response, MTT-based cell viability analyses of Ctx-treated MCF10A/HER2 cells, the $\mathrm{IC}_{50}$ value for viability was not reached, even at doses as high as $200 \mu \mathrm{g} / \mathrm{ml}$ (Fig. 1, right).

Acquired (secondary) resistance to Ctx is accompanied by transcriptional upregulation of HER 2 overexpression in squamous cell carcinoma cells. Since predictors of efficacy in non-small cell lung carcinomas, in which KRAS mutations do not predict Ctx efficacy, and in head and neck squamous cell carcinomas with intact $K R A S$ signaling are also lacking, we propose that our recently established pre-clinical models of EGFR geneamplified wild-type KRAS A431 squamous cell carcinoma, in which cells are chronically adapted to grow in the presence of Ctx (10-12), could be used to determine if the HER2 expression status is significantly altered during and/or after treatment with Ctx. We first performed real-time (kinetic) RT-PCR analyses to quantitatively track the transcriptional evolution of the entire family of HER receptors [i.e., EGFR (HER1), HER2, HER3, and HER4] during Ctx-induced deactivation of the EGFR/RAS/ MAPK pathway. Analyzing the changes in the gene expression profiles of the HER1/-2/-3/-4 receptors during acute (i.e., $6 \mathrm{~h}$ ) treatment with Ctx revealed that Ctx treatment significantly increased HER2 mRNA expression by $\sim 3$-fold (Fig. 2A, left). An equivalent upregulation of HER 3 mRNA (3-fold) was observed in response to acutely added Ctx. The expression status of EGFR (HER1) at the mRNA level remained unaltered following Ctx exposure. HER4 expression was not detectable 
A
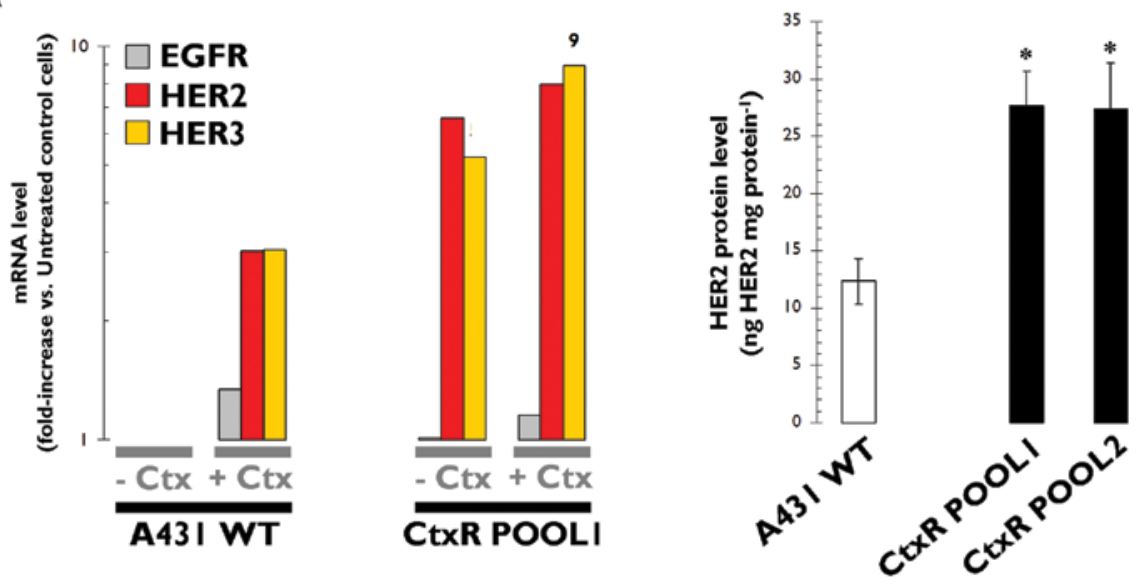

B
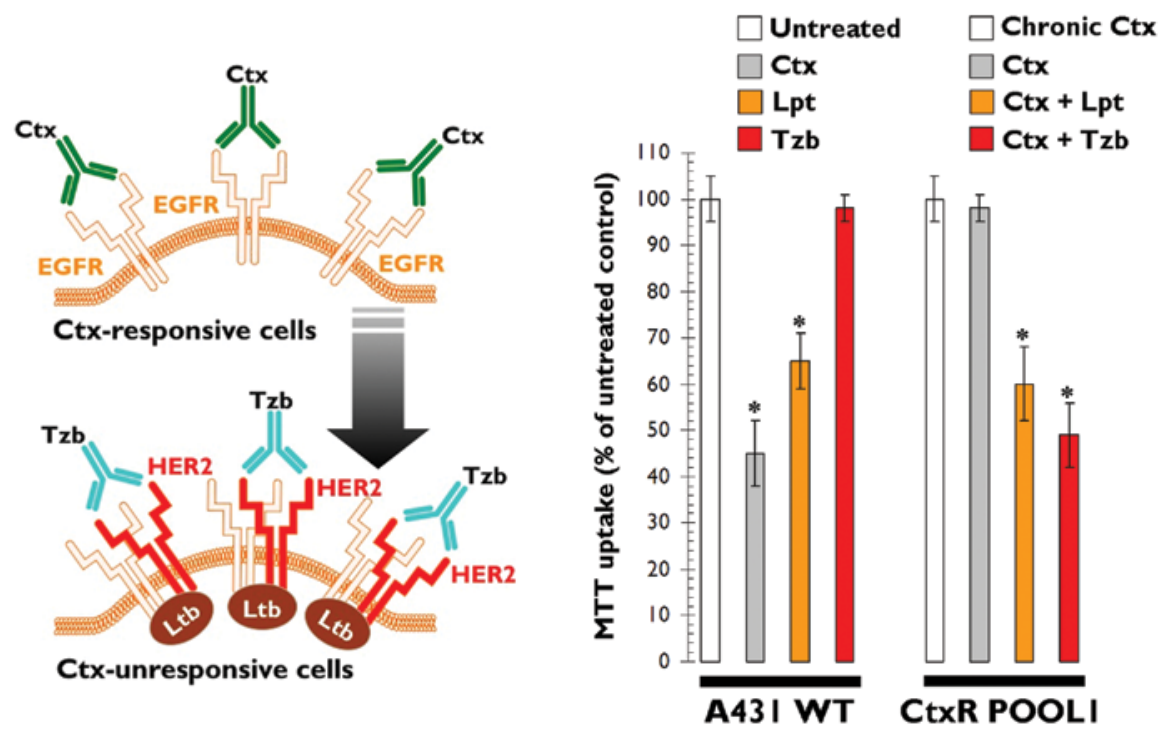

Figure 2. (A) HER2 expression is transcriptionally upregulated in SCC cells with acquired resistance to Ctx. Image shows the mean fold changes (columns) in expression of the target genes relative to internal control gene $6 \mathrm{~h}$ after treatment with $100 \mu \mathrm{g} / \mathrm{ml} \mathrm{Ctx}$ calculated by using the $2^{-\Delta \Delta C_{t}}$ method as described in Materials and methods. (B) Acquired resistance to Ctx in SCC cells is reversed by the mono-HER2 inhibitor trastuzumab. Ctx-responsive A431 parental cells and their derivatives with acquired resistance to Ctx were cultured for 5 days in the absence or presence of Ctx (100 $\mu \mathrm{g} / \mathrm{ml})$, lapatinib (Lpt; $1 \mu \mathrm{mol} / \mathrm{l})$ and/or trastuzumab (Tzb; $100 \mu \mathrm{g} / \mathrm{ml}$ ), as specified. Cell viability was measured with the MTT uptake assay and expressed as \% of untreated cell population (=100\%). Results are means (columns) and 95\% confidence intervals (bars) of five independent experiments made in triplicate. Statistically significant differences (onefactor ANOVA analysis) between MTT uptakes in treated and untreated control cells are shown (" $\mathrm{P}<0.01)$. Statistical tests were two-sided.

by RT-PCR in A431 cells (data not shown). Using pre-clinical models of acquired Ctx resistance, which were developed in our laboratory by chronically adapting A431 cells to grow in the continuous presence of Ctx for a minimum of 6 months (10-12), we observed that mRNA levels of HER2 and HER3 were drastically upregulated by $\sim 7$ - and 8 -fold, respectively, in one of the Ctx-unresponsive pooled A431 cell populations (Fig. 2A, left). Upregulations in the HER2 and HER3 mRNA levels of $>4$-fold were detected in a second pooled population of Ctx-resistant A431 cells (data not shown). Notably, this upregulation in the HER2 and HER3 mRNA levels remained unaltered in response to Ctx challenge (Fig. 2A, left).

Co-exposure to the anti-HER 2 monoclonal antibody trastuzumab reverses acquired resistance to Ctx in squamous cell carcinoma cells. ELISA-based determination of HER2 expression confirmed that the HER2 protein is significantly upregulated in A431 cells once resistance to Ctx is acquired (Fig. 2A, right). However, ELISA experiments showed that the changes in expression of the HER2 protein do not match the more prominent upregulation of HER2 at the mRNA level. Although the ultimate molecular mechanism underlying this lack of correlation between the increase in HER2 mRNA levels (up to 7-fold) and the smaller increase in HER2 protein expression $(\sim 2$-fold) is unclear, we evaluated the functional activity of the transcriptionally upregulated HER2 protein and its causal relationship with the acquisition of resistance to Ctx (Fig. 2B, left). When we added the dual HER1/HER2 TKI lapatinib (Tykerb) to the culture medium of Ctx-resistant A431 cells already containing cetuximab (to simulate the treatment progression used in the clinic), we observed that Ctx-resistant A431 squamous cancer cells not only retained 
lapatinib sensitivity but were slightly more sensitive than Ctx-naïve A431 parental cells to the growth-inhibitory effects of lapatinib (Fig. 2B, right). This suggests an increase in HER2 signaling in Ctx-resistant cells, which was confirmed using the mono-HER2 inhibitor trastuzumab (Herceptin). While HER1 (EGFR)-dependent A431 parental cells were also refractory to trastuzumab-induced blockade of HER2, a reduction of $\sim 50 \%$ in MTT dye uptake, which correlates with cell viability, was observed in trastuzumab-treated Ctx-resistant A431 cells (Fig. 2B, right). We believe that these qualitative and quantitative differences in the antitumor efficacy of trastuzumab are a direct consequence of the pro-survival function driven by the transcriptional upregulation of HER2 in EGFR-positive A431 cells that have acquired resistance to $\mathrm{Ctx}$.

\section{Discussion}

Our current findings reveal that EGFR-positive, $K R A S$ wildtype cancer cells with acquired resistance to $\mathrm{Ctx}$ can become addicted to HER2-driven signaling and can therefore benefit from subsequent treatment with the anti-HER2 monoclonal antibody trastuzumab, providing a rationale for the use of HER2-targeted therapies in the presence of Ctx resistance. This phenomenon should be taken into account during the design of studies addressing the role of HER2 in inherent (primary) and acquired (secondary) Ctx resistance and for developing strategies for testing Ctx in combination with HER2 inhibitors such as trastuzumab in the absence of HER2 amplification. The benefits of adjuvant trastuzumab may not be limited to patients with HER2 gene amplification $(13,14)$, and trastuzumab efficiently triggers receptor-enhanced chemosensitivity (REC) when combined with chemotherapy in cell lines with low-to-intermediate HER2 expression, i.e., without HER2 overexpression $(15,16)$. Moreover, trastuzumab may work by targeting cells with tumor-propagating properties; such cells, notably, are enriched in Ctx-resistant pooled populations of A431 cells (11), which have increased levels of HER2 protein compared with the bulk cell population, in which HER2 gene amplification is unmodified (17-19). Although this is an in vitro study, if our current data were to be validated with clinical samples, the threshold values for HER2 positivity may need to be redefined for Ctx-refractory patients with wild-type $K R A S$ that might benefit from receiving a $\mathrm{Ctx} /$ trastuzumab combination. Theoretically, the guidelines regarding trastuzumab administration would then need to include more Ctx-refractory cases than those selected based solely on the presence of HER2 gene amplification.

\section{Acknowledgements}

The work in the laboratory of Javier A. Menendez was supported by the Instituto de Salud Carlos III (Ministerio de Sanidad y Consumo, Fondo de Investigación Sanitaria (FIS), Spain, Grants CP05-00090 and PI06-0778 and RD06-00200028), the Fundación Científica de la Asociación Española Contra el Cáncer (AECC, Spain), and by the Ministerio de Ciencia e Innovación (SAF2009-11579, Plan Nacional de I+D+ I, MICINN, Spain). Alejandro Vazquez-Martin is the recipient of a Sara Borrell post-doctoral contract (CD08/00283, Ministerio de Sanidad y Consumo, Fondo de Investigación
Sanitaria -FIS-, Spain). Sílvia Cufí is the recipient of a Research Fellowship (Formación de Personal Investigador, FPI) from the Ministerio de Ciencia e Innovación (MICINN, Spain). Cristina Oliveras-Ferraros received a research salary from a Grant Award by the 'Fundacion Salud 2000', which is promoted by Merck Serono (Madrid, Spain).

\section{References}

1. Yonesaka K, Zejnullahu K, Okamoto I, Satoh T, Cappuzzo F, Souglakos J, Ercan D, Rogers A, Roncalli M, Takeda M, et al: Activation of ERBB2 signaling causes resistance to the EGFRdirected therapeutic antibody cetuximab. Sci Transl Med 3: 99ra86, 2011.

2. Quesnelle KM and Grandis JR: Dual kinase inhibition of EGFR and HER2 overcomes resistance to cetuximab in a novel in vivo model of acquired cetuximab resistance. Clin Cancer Res 17: 5935-5944, 2011.

3. Bertotti A, Migliardi G, Galimi F, Sassi F, Torti D, Isella C, Corà D, Di Nicolantonio F, Buscarino M, Petti C, et al: A molecularly annotated platform of patient-derived xenografts ('xenopatients') was exploited for identifying novel mechanisms of resistance to cetuximab-resistant colorectal cancer. Cancer Discov 1: 508-523, 2011.

4. Ciardiello F and Normanno N: HER2 signaling and resistance to the anti-EGFR monoclonal antibody cetuximab: a further step toward personalized medicine for patients with colorectal cancer. Cancer Discov 1: 472-474, 2011.

5. Ciardiello F, Gottardis M, Basolo F, Pepe S, Normanno N, Dickson RB, Bianco AR and Salomon DS: Additive effects of c-erbB-2, c-Ha-ras, and transforming growth factor-alpha genes on in vitro transformation of human mammary epithelial cells. Mol Carcinog 6: 43-52, 1992.

6. Giunciuglio D, Culty M, Fassina G, Masiello L, Melchiori A, Paglialunga $\mathrm{G}$, Arand $\mathrm{G}$, Ciardiello F, Basolo $\mathrm{F}$ and Thompson EW: Invasive phenotype of MCF10A cells overexpressing c-Ha-ras and c-erbB-2 oncogenes. Int J Cancer 63: 815-822, 1995

7. Ciardiello F, Caputo R, Pomatico G, De Laurentiis M, De Placido S, Bianco AR and Tortora G: Resistance to taxanes is induced by c-erbB-2 overexpression in human MCF-10A mammary epithelial cells and is blocked by combined treatment with an antisense oligonucleotide targeting type I protein kinase $\mathrm{A}$. Int J Cancer 85: 710-715, 2000.

8. Menendez JA, Vazquez-Martin A, Oliveras-Ferraros C, GarciaVillalba R, Carrasco-Pancorbo A, Fernandez-Gutierrez A and Segura-Carretero A: Extra-virgin olive oil polyphenols inhibit HER2 (erbB-2)-induced malignant transformation in human breast epithelial cells: relationship between the chemical structures of extra-virgin olive oil secoiridoids and lignans and their inhibitory activities on the tyrosine kinase activity of HER2. Int J Oncol 34: 43-51, 2009.

9. Konecny GE, Pegram MD, Venkatesan N, Finn R, Yang G, Rahmeh M, Untch M, Rusnak DW, Spehar G, Mullin RJ, et al: Activity of the dual kinase inhibitor lapatinib (GW572016) against HER-2-overexpressing and trastuzumab-treated breast cancer cells. Cancer Res 66: 1630-1639, 2006.

10. Oliveras-Ferraros C, Vall-Llovera AM, Salip DC, VazquezMartin A, Cufí S, Queralt B, Martin-Castillo B, Brunet J, de Llorens R and Menendez JA: Evolution of the predictive markers amphiregulin and epiregulin mRNAs during long-term cetuximab treatment of KRAS wild-type tumor cells. Invest New Drugs 30: 846-852, 2012.

11. Oliveras-Ferraros C, Vazquez-Martin A, Cufí S, Queralt B, Báez L, Guardeño R, Hernández-Yagüe X, Martin-Castillo B, Brunet $J$ and Menendez JA: Stem cell property epithelial-tomesenchymal transition is a core transcriptional network for predicting cetuximab (Erbitux ${ }^{\mathrm{TM}}$ ) efficacy in KRAS wild-type tumor cells. J Cell Biochem 112: 10-29, 2011.

12. Oliveras-Ferraros C, Vazquez-Martin A, Queralt B, Adrados M, Ortiz R, Cufí S, Hernández-Yagüe X, Guardeño R, Báez L, Martin-Castillo B, et al: Interferon/STAT1 and neuregulin signaling pathways are exploratory biomarkers of cetuximab (Erbitux ${ }^{\circledR}$ ) efficacy in KRAS wild-type squamous carcinomas: a pathway-based analysis of whole human-genome microarray data from cetuximab-adapted tumor cell-line models. Int J Oncol 39: 1455-1479, 2011 
13. Paik S, Kim $\mathrm{C}$ and Wolmark N: HER 2 status and benefit from adjuvant trastuzumab in breast cancer. $\mathrm{N}$ Engl J Med 358: 1409-1411, 2008

14. Perez EA, Reinholz MM,Hillman DW, Tenner KS, Schroeder MJ, Davidson NE, Martino S, Sledge GW, Harris LN, Gralow JR, et al: HER 2 and chromosome 17 effect on patient outcome in the N9831 adjuvant trastuzumab trial. J Clin Oncol 28: 4307-4315, 2010.

15. Arteaga CL: Can trastuzumab be effective against tumors with low HER2/Neu (ErbB2) receptors? J Clin Oncol 24: 3722-3725, 2006.

16. Menendez JA, Mehmi I and Lupu R: Trastuzumab in combination with heregulin-activated Her-2 (erbB-2) triggers a receptorenhanced chemosensitivity effect in the absence of Her-2 overexpression. J Clin Oncol 24: 3735-3746, 2006.

17. Korkaya $\mathrm{H}$ and Wicha MS: HER-2, notch, and breast cancer stem cells: Targeting an axis of evil. Clin Cancer Res 15: 1845-1847, 2009.

18. Magnifico A, Albano L, Campaner S, Delia D, Castiglioni F, Gasparini P, Sozzi G, Fontanella E, Menard S and Tagliabue E: Tumor-initiating cells of HER2-positive carcinoma cell lines express the highest oncoprotein levels and are sensitive to trastuzumab. Clin Cancer Res 15: 2010-2021, 2009.
19. Vazquez-Martin A, Oliveras-Ferraros C, Del Barco S, MartinCastillo B and Menendez JA: The anti-diabetic drug metformin suppresses self-renewal and proliferation of trastuzumabresistant tumor-initiating breast cancer stem cells. Breast Cancer Res Treat 126: 355-364, 2011.

20. Ferrer-Soler L, Vazquez-Martin A, Brunet J, Menendez JA, De Llorens R and Colomer R: An update of the mechanisms of resistance to EGFR-tyrosine kinase inhibitors in breast cancer: Gefitinib (Iressa)-induced changes in the expression and nucleocytoplasmic trafficking of HER-ligands (Review). Int J Mol Med 20: 3-10, 2007.

21. Ritter CA, Perez-Torres M, Rinehart C, Guix M, Dugger T, Engelman JA and Arteaga CL: Human breast cancer cells selected for resistance to trastuzumab in vivo overexpress epidermal growth factor receptor and ErbB ligands and remain dependent on the ErbB receptor network. Clin Cancer Res 13: 4909-4919, 2007. 